\title{
A Hybrid Method Based on the Iterative Fourier Transform and the Differential Evolution for Pattern Synthesis of Sparse Linear Arrays
}

\author{
Xin-Kuan Wang $(\mathbb{D}$ and Gui-Bao Wang $\mathbb{D}$ \\ School of Physics and Telecommunication Engineering, Shaanxi University of Technology, Hanzhong 723000, China \\ Correspondence should be addressed to Xin-Kuan Wang; wxkuan@snut.edu.cn
}

Received 18 April 2018; Accepted 12 June 2018; Published 5 July 2018

Academic Editor: Francesco D'Agostino

Copyright (c) 2018 Xin-Kuan Wang and Gui-Bao Wang. This is an open access article distributed under the Creative Commons Attribution License, which permits unrestricted use, distribution, and reproduction in any medium, provided the original work is properly cited.

\begin{abstract}
A hybrid method that combines the iterative Fourier transform (IFT) and the algorithm of differential evolution (DE) is proposed to address the synthesis of low sidelobe sparse linear array (SLA) including many elements. Firstly, a thinned linear array (TLA) with the lattices spaced at half wavelength is obtained by the IFT. Then, for the elements of the TLA whose left or right spacing are greater than half wavelength, their placements are selected as the candidates which will be further optimized by the DE, as long as the interelement spacing is not less than half wavelength. Consequently, the convergence pressure of DE is greatly alleviated for the reason that the selected elements only accounts for a small part of the total. Therefore, the SLA with improved sidelobe performance can be obtained at relative low hardware cost. Several numerical instances confirmed the effectiveness of the proposed method.
\end{abstract}

\section{Introduction}

In some antenna applications, we focus the antenna array with narrow beamwidth to increase the resolution while allow sacrificing some antenna gain, so a certain percentage of the elements can be removed from a periodic, fully populated array. This is commonly known as thinned array (TA). For the advantages in reducing weight, power, and cost, TA had been used in satellite communications, ground-based radars, radio astronomy, and so on [1]. Over the past few decades, a number of tools, such as the genetic algorithm (GA) [2-4], the ant colony optimization (ACO) [5], the particle swarm optimization (PSO) [6], the biogeography-based optimization (BBO) [7], IFT [8-10], and the almost difference set- (ADS-) based approach [11], had been employed for TA synthesizing. In particular, the IFT [9] and ADS [11] had been confirmed very effective for thinning large planar arrays.

Another kind of structure analogous to TA, which has the elements arbitrarily located within the antenna aperture and thereby provides more degree of freedom to achieve sidelobe suppression, is called sparse array (SA). Unfortunately, the synthesis for SA refers to a question of multiple constraints including the minimum element spacing, the antenna aperture, and the number of elements. Therefore, the use of stochastic optimization algorithms such as the simulated annealing [12], PSO [13], DE [14, 15], and GA [16] is not a computationally efficient way for the synthesis of the SA including many elements. During the past few years, the deterministic approaches, such as the IFT [8-10], the methodology by means of density taper [17], had been proposed. Most of this kind of methods require a priori estimate for the array performance as well as a predefined number of elements within a given aperture size; therefore, they cannot guarantee the array fitting desired power patterns with maximally reduced number of elements. Recently, a noniterative method for linear array based on the matrix pencil method (MPM) [18] is proposed and confirmed to be effective and computationally efficient in synthesizing the massively sparse arrays. More notably, the Bayesian compressive sensing (BCS) had been employed [19] to address the same issue. The reports in $[19,20]$ shown that the BCS-based approach 


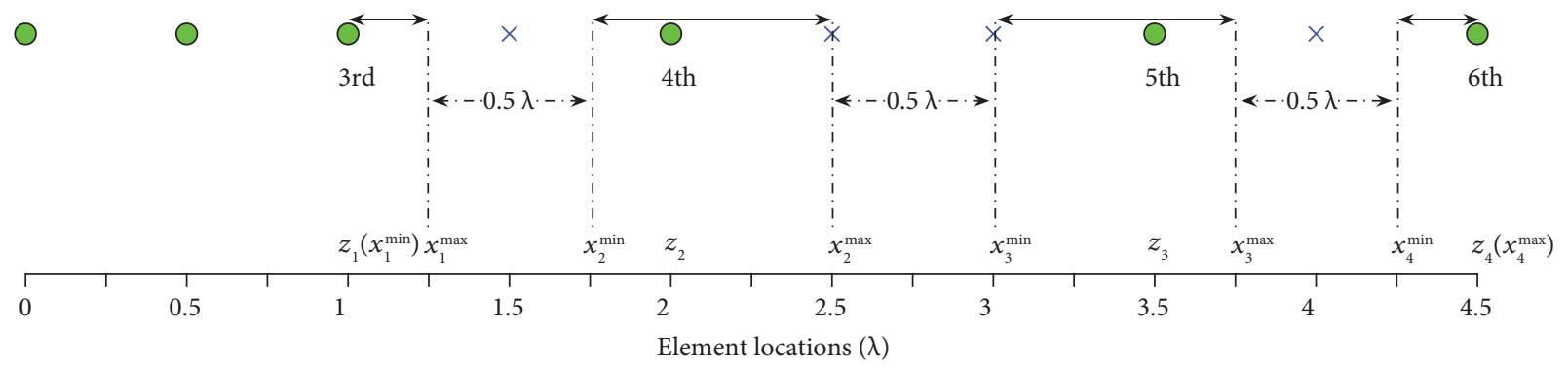

Figure 1: A thinned array to illustrate the way how to select the element locations that need to be optimized.

allows the design of linear arrangement fitting desired power patterns with at least $35 \%$ elements reduced comparing to the regular array configuration. Furthermore, in order to synthesize the asymmetrical shaped beams, an advanced term of the BCS is applied in [21], which could deal with the SLA matching arbitrary reference patterns.

In this manuscript, the synthesis of uniform-amplitude sparse linear array with many elements is considered. Naturally, the use of the stochastic method, typically as DE, always involves a large set of parameters and thereby requires high computational resources. To ease the impact, we propose a hybrid method that combines the IFT and DE (IFT-DE) to address the issue. The method is based on DE with the individual parameters constituted by the element locations. Differently, unlike the conventional DE in which all the individual parameters are randomly initialized, for the assumption that the element spacing is not less than 0.5 $\lambda$ ( $\lambda$ denotes the wavelength), most element locations of this method are predetermined by a uniform excited TLA with the lattices spaced at $0.5 \lambda$ that obtained by the IFT, the first step of the IFT-DE. Consequently, just a limited number of individual parameters that remain for $\mathrm{DE}$ are to be optimized. This will effectively narrow the solution space and thereby efficiently accelerate the convergence speed of DE.

The manuscript is mainly organized as follows. Section 2 describes the formulation of the proposed method. Section 3 provides a set of numerical examples to validate the effectiveness of the IFT-DE. Finally, the conclusion of this work is summarized in Section 4.

\section{Formulation of the Proposed Method}

Consider a linear array with $N$ isotropic elements. The array factor can be expressed as follows [8]:

$$
A F(u)=\sum_{n=0}^{N-1} A_{n} e^{j k n d u}
$$

where $A_{n}$ represents the $n$th element excitation, and all the excitations are labeled as the collection $\left\{A_{n}\right\} . k$ is the wavelength, and $d$ is the element spacing. $u=\sin \theta$, where $\theta$ denotes the angle measured from the broadside of the array.

The IFT in the proposed method is the same as that used in [10], which is a slight modification form to that presented in [8]. Therefore, the adaptively changed filling factor can be written as

$$
f=1-q \Delta f, \quad q=1,2, \ldots, Q,
$$

where $\Delta f$ refers to the step size when $f$ changes, $q$ is the iteration number with $Q$ representing its maxima. When $f$ reaches its target value $f_{0}, Q$ can be written as

$$
Q=\frac{1-f}{\Delta f} .
$$

Accordingly, a single trial of the IFT-DE can be divided into three steps, as are in turn described below.

Step 1. Perform the IFT to get a thinned linear array [8-10].

(1) Randomly initialize the element excitations $\left\{A_{n}\right\}$ over the $N$ lattices with probability of 0.9 equal to one and the rest equal to zero.

(2) Compute the array factor $A F(u)$ from $\left\{A_{n}\right\}$ through $K$ points IFFT with $K>2 N$.

(3) Force the values in the sidelobe region of $A F(u)$ to match the predefined value that is obtained by trial and error.

(4) Compute $\left\{A_{n}\right\}$ from $A F(u)$ through $K$ points FFT.

(5) Truncate the $K$ samples of $\left\{A_{n}\right\}$ to $N$ samples that coincide with the total number of lattices.

(6) For $\left\{A_{n}\right\}$, set the $N \cdot f$ samples which have higher amplitudes to be equal to 1 , while others equal to 0 .

(7) Decrease $f$ with step size of $\Delta f$ so that in each iteration cycle, just one element is removed for asymmetrical array while in the opposite, two elements located in symmetrical positions are removed.

(8) Repeat steps (2)-(7) until $f$ arrives at $f_{0}$, then the iteration is terminated and a thinned array with filling factor of $f_{0}$ is obtained.

Step 2. Select the element locations that need to be optimized. For the above thinned array, select the elements whose left or right spacing are greater than $0.5 \lambda$, and label their initial locations as the set $\left\{z_{l}, l=1,2, \ldots, L\right\}$, where $L$ refers to the maximum number. Figure 1 illustrates a thinned 
array including six elements with the lattices spaced at $0.5 \lambda$. The symbols of solid circle indicate the activated elements, while the symbols " $x$ " describe the other way (Figure 1). It can be seen that the elements numbered in 3rd, 4th, 5th, and 6th, whose initial locations are in turn recorded by $z_{1}, z_{2}, z_{3}$, and $z_{4}$, are deemed to meet the aforementioned selection rule.

Step 3. The optimization for the selected element locations using DE.

(1) Determine the lower and upper bounds for locations of the selected elements.

Assume that a total of $L$ element locations $x_{l}, l=1, \ldots, L$ need to be optimized with $z_{l}, l=1, \ldots, L$ the initial value, and the lower and upper bounds for $x_{l}$ are, respectively, indicated by $x_{l}^{\min }$ and $x_{l}^{\max }, l=1, \ldots, L$. For the constraint that the minimum element spacing is not less than $0.5 \lambda$, the values of $x_{l}^{\min }$ and $x_{l}^{\max }$ are set to be determined as follows:

$$
\begin{gathered}
x_{l}^{\max }-z_{l}=z_{l+1}-x_{l+1}^{\min }, \\
x_{l+1}^{\min }-x_{l}^{\max }=0.5 \lambda, \\
\quad l=1,2, \ldots, L-1 .
\end{gathered}
$$

It depicts that the maximum range of the adjacent elements moving in the opposite direction is set equal, and the minimum distance after the movement is $0.5 \lambda$. To illustrate the above restriction, still in Figure 1 as an example, the location ranges (described by the double arrows) for the elements numbered in $3 \mathrm{rd}, 4$ th, 5 th, and 6 th are given by the following set of inequalities

$$
\begin{gathered}
1.0 \lambda \leq x_{1} \leq 1.25 \lambda \\
1.75 \lambda \leq x_{2} \leq 2.5 \lambda \\
3.0 \lambda \leq x_{3} \leq 3.75 \lambda \\
4.25 \lambda \leq x_{4} \leq 4.5 \lambda
\end{gathered}
$$

\section{(2) Perform DE.}

A conventional DE similar to that presented in [22] is used to find the SLA with minimum peak sidelobe level (PSLL), where the mutation strategy of $\mathrm{DE} / \mathrm{rand} / 1$, as well as the operator of binomial crossover, is adopted. Once the overall steps are finished, the best fitness value so far is retained, and the method will proceed to the next trial until it reaches the maxima.

\section{Numerical Examples}

The proposed method is applied to the design cases similar to that taken from $[3,5,8]$. Therefore, the synthesis using the IFT-DE refers to firstly obtaining a TLA with different filling factors based on a total of 200 or 100 lattices spaced at $0.5 \lambda$. In each numerical instance, the IFT-DE is run for 100 independent trials. The sampling points of FFT are equal to
4096, and the initial parameters of DE, including the maxima of evolutionary generation, the population size, the scale factor, and the crossover probability, are equal to $20,20,0.7$, and 0.9 , respectively.

3.1. Comparing with the Reports Obtained by the IFT [8]. We consider synthesizing a symmetrical/asymmetrical SLA that is originally based on 200 lattices, symmetrical/asymmetrical TLA with different filling factors. The first case refers to synthesizing a symmetrical SLA through a symmetrical TLA with filling factor of $66 \%$. The fitness convergence curve indicates that the optimal fitness value among 100 trials of IFT-DE is obtained at trial number 94 (Figure 2(a). The far-field pattern, as is described in Figure 2(b), has the PSLL of $-24.59 \mathrm{~dB}$, about $1.73 \mathrm{~dB}$ sidelobe reduction compared with the report presented in [8], while the $3 \mathrm{~dB}$ beamwidth almost remained unchanged. To demonstrate the implementation process of the IFT-DE, Figure 3 gives the element distribution of the thinned linear array originally obtained by the IFT, the first step of this method at the same trial. The plane structure, started from the first row to the tenth, and each row from the left to right, describes in turn the TLA's element locations, where the "solid squares" indicate the activated elements whose locations need to be optimized. As we can see, there are a total of 38 elements whose locations remain to be optimized among 132 candidates, and these locations are confined in some lattices. Furthermore, when the method is thoroughly performed, the element distribution of the SLA can be described by the plane structure in Figure 4 . We can see that the positions of the selected elements are no longer confined in the lattices but randomly located around them. However, the unselected elements are still located in their initial lattices.

Similarly, as far as a symmetrical TLA with a filling factor of $77 \%$ is originally concerned, the far-field pattern of the best symmetrical SLA among 100 trials of IFT-DE shows $1 \mathrm{~dB}$ PSLL reduction compared with the report presented in [8], while the $3 \mathrm{~dB}$ beamwidth is almost unchanged. The third case considers an asymmetrical TLA with a filling factor equal to $39 \%$. Figure 5(a) shows that the best sparse array is obtained at trial number 68 with the fitness value equal to $-20.38 \mathrm{~dB}$, about $3.0 \mathrm{~dB}$ lower than PSLL reported in [8]. However, the far-field pattern, as is depicted in Figure 5(b), only shows a beam-broaden about 0.254 degrees. The element locations of this SLA are described in Figure 6. It indicates that a total of 33 elements had their locations being readjusted among the 78 activated elements.

The fourth example assumes an asymmetrical TLA that is $69.5 \%$ filled. The obtained SLA shows about $1.16 \mathrm{~dB}$ PSLL decrement with no sacrifice of beamwidth, as is compared to the value presented in [8].

3.2. Comparing with the Reports Obtained by GA and ACO $[3,5]$. We first consider synthesizing a low sidelobe symmetrical SLA based on 100 lattices, symmetrical TLA with a filling factor of $80 \%$. The PSLL of the sparse array obtained by IFT-DE is equal to $-20.52 \mathrm{~dB}$, which is $1.7 \mathrm{~dB}$ lower than the reports using ACO [5]. More notably, when the filling factor decreased to $78 \%$ and $76 \%$, the SLA 


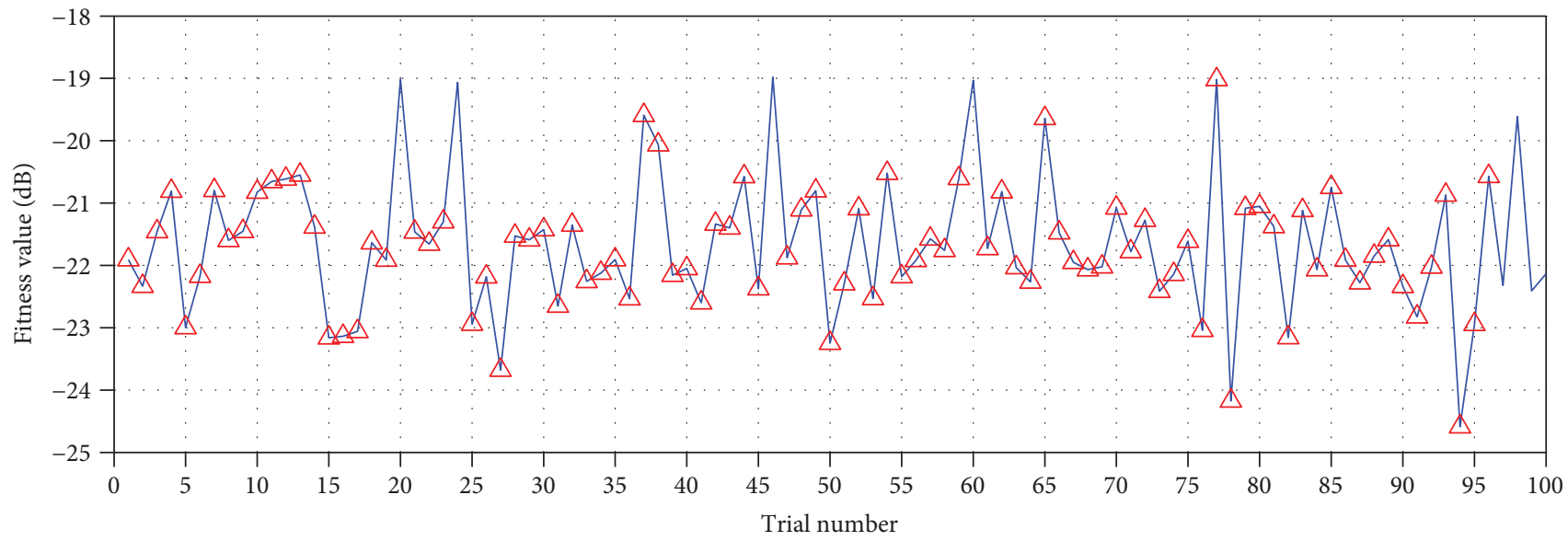

(a)

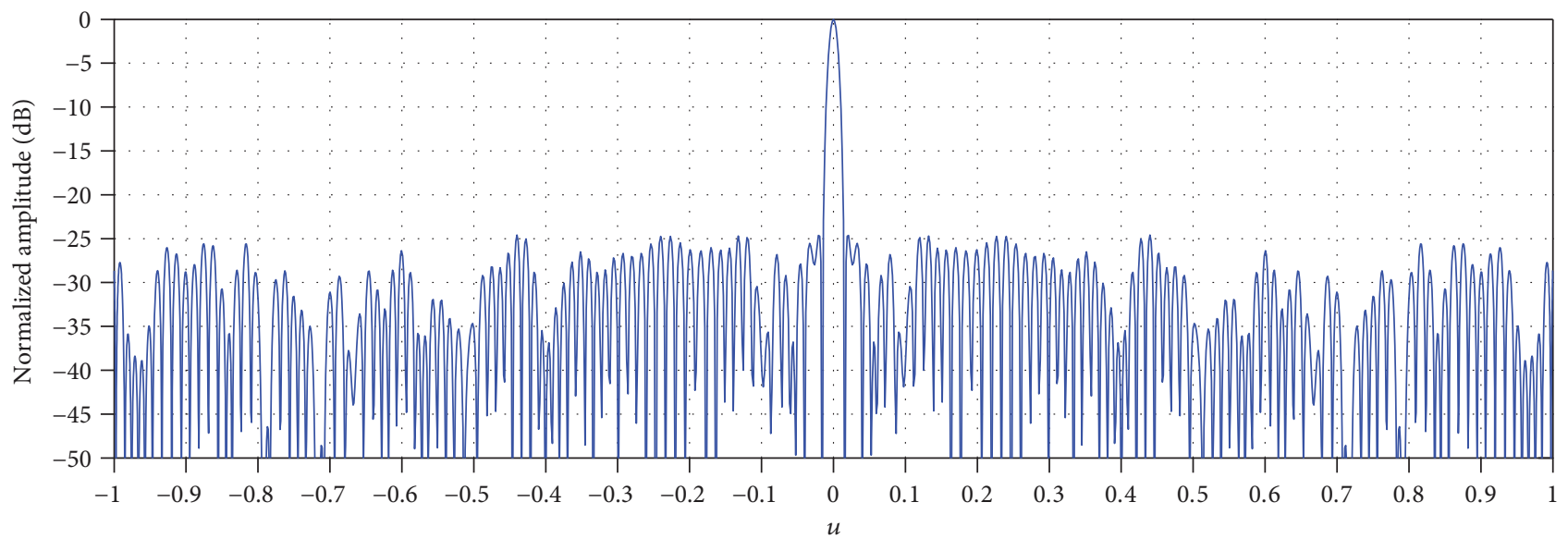

(b)

FIgURE 2: The sparse linear array obtained by the IFT-DE based on a symmetrical TLA with a filling factor of $66 \%$. (a) The convergence curve of the fitness value. (b) The optimal far-field pattern among 100 trials.

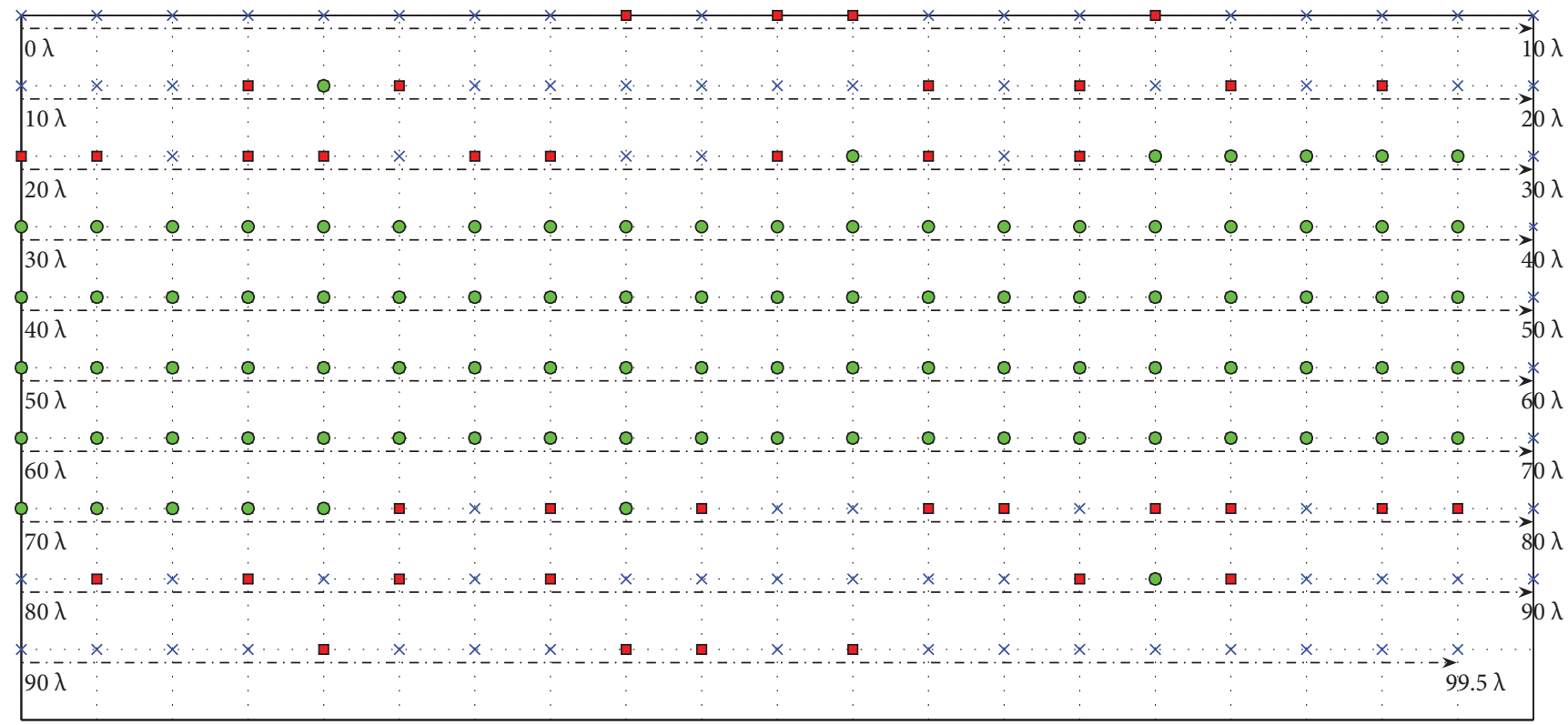

FIgURE 3: The element locations of the symmetrical TLA with a filling factor of $66 \%$ obtained by the IFT. 


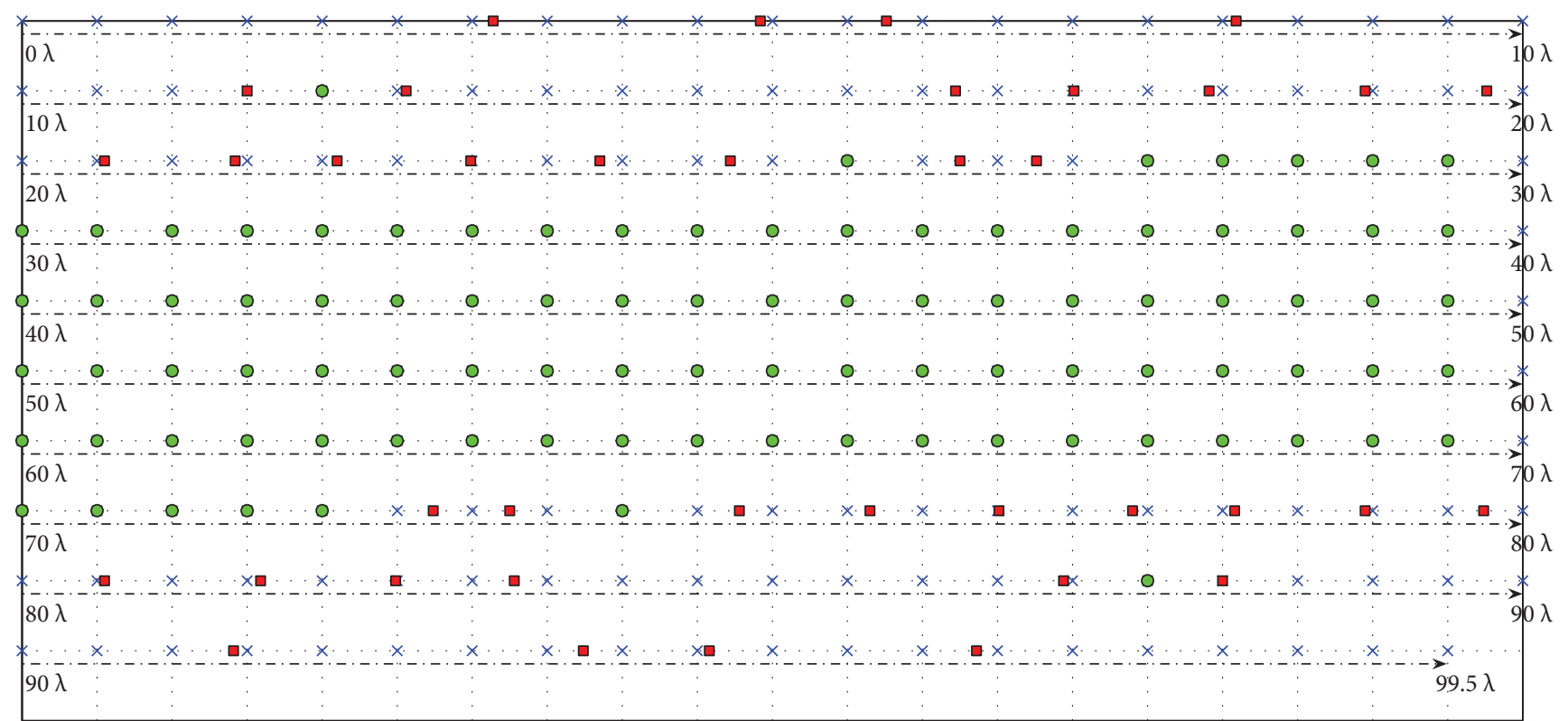

FIGURE 4: The element locations of the sparse linear array obtained by the IFT-DE based on the TLA described in Figure 3.

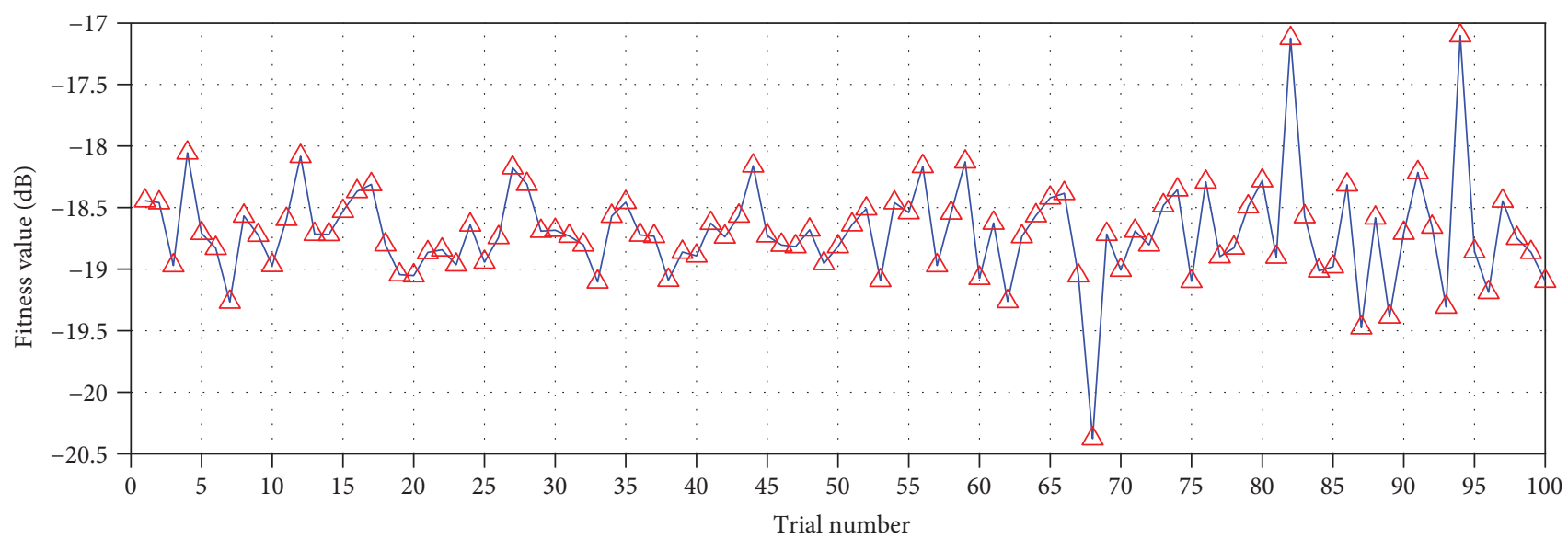

(a)

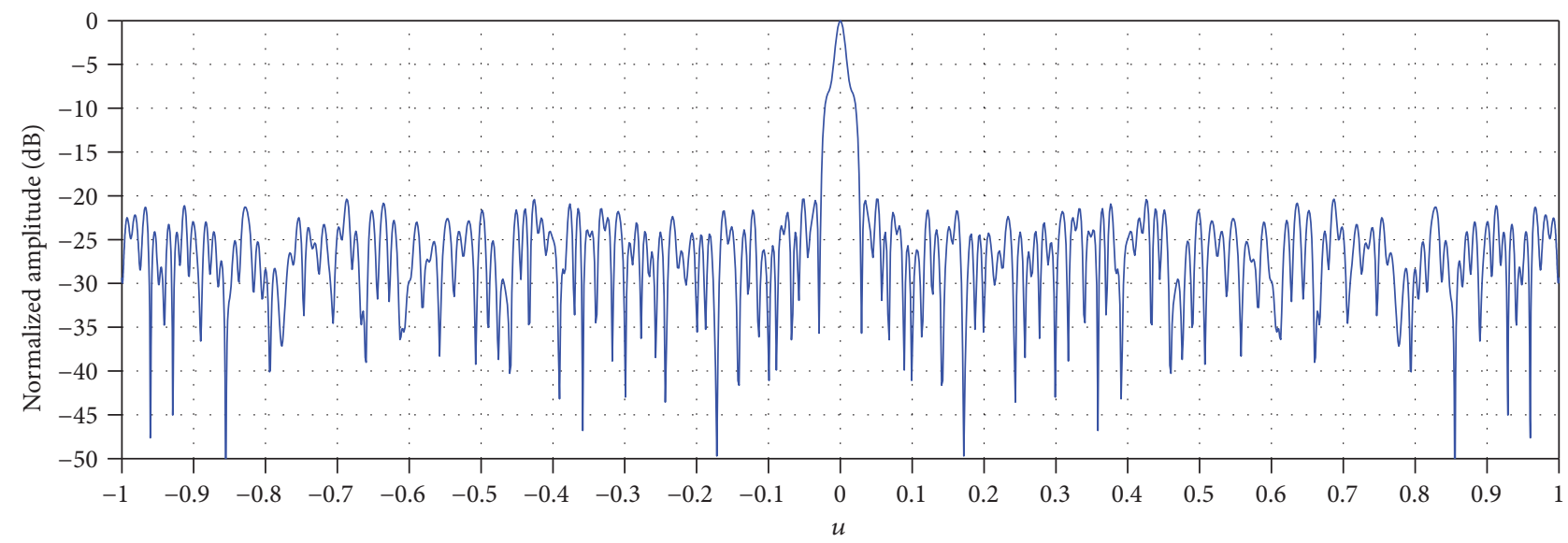

(b)

FIGURE 5: The sparse linear array obtained by the IFT-DE based on an asymmetrical TLA with a filling factor of 39\%. (a) The convergence curve of the fitness value. (b) The optimal far-field pattern among 100 trials. 


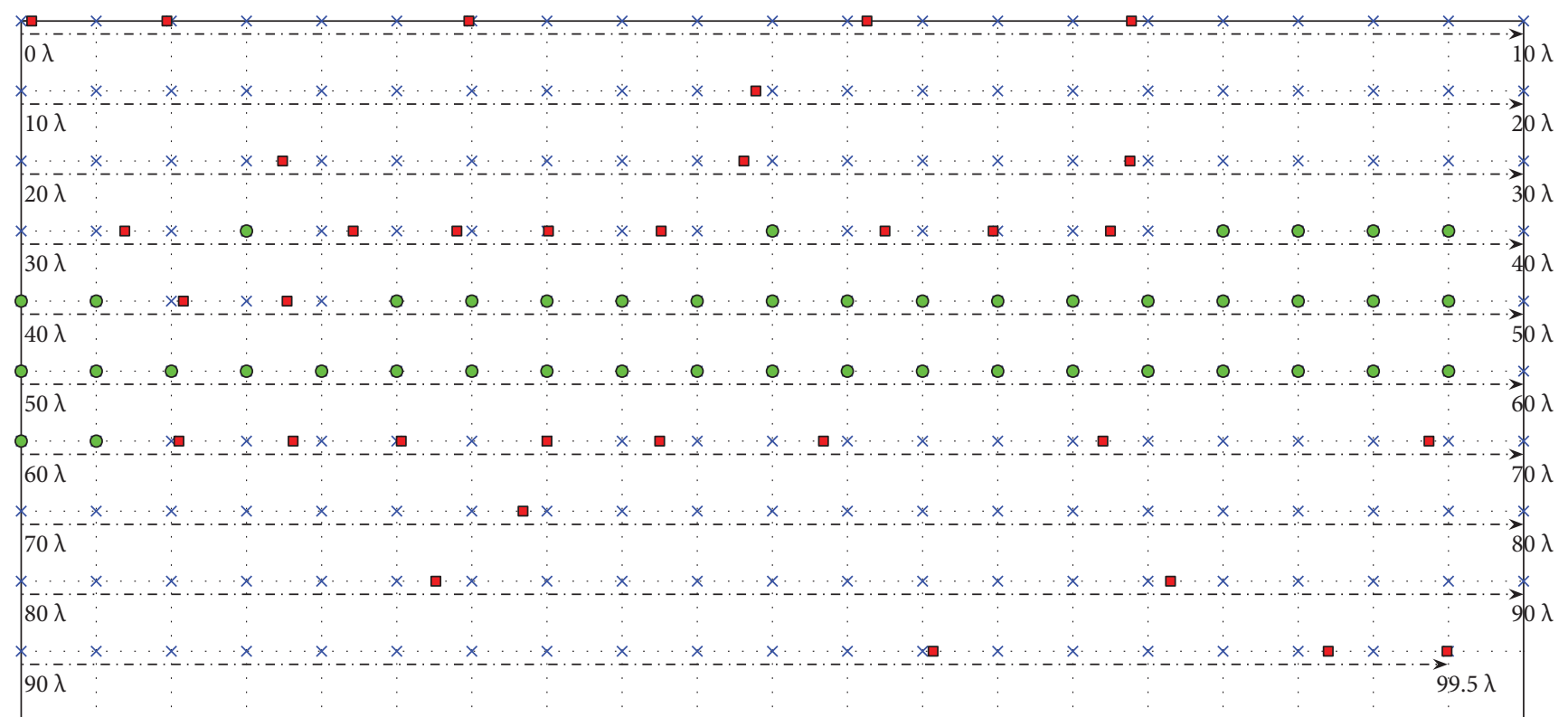

FIgURE 6: The element locations of the sparse linear array obtained by the IFT-DE based on an asymmetrical TLA with a filling factor of $39 \%$.

TABLE 1: Comparative synthesizing results by using the IFT-DE and some published tools.

\begin{tabular}{|c|c|c|c|c|c|}
\hline \multirow{2}{*}{ The structure of the SLA } & \multirow{2}{*}{$\begin{array}{c}\text { Filling factor of the TLA originally } \\
\text { obtained by the IFT (\%) }\end{array}$} & \multicolumn{2}{|c|}{ PSLL (dB) } & \multicolumn{2}{|c|}{$3 \mathrm{~dB}$ beamwidth (degree) } \\
\hline & & IFT-DE & IFT [8] & IFT-DE & IFT [8] \\
\hline \multirow{2}{*}{ Symmetrical } & $77(N=200)$, symmetrical & -24.04 & -22.92 & 0.58 & 0.588 \\
\hline & $66(N=200)$, symmetrical & -24.59 & -22.86 & 0.68 & 0.685 \\
\hline \multirow{2}{*}{ Asymmetrical } & $69.5(N=200)$, asymmetrical & -25.46 & -24.3 & 0.64 & 0.643 \\
\hline & $39(N=200)$, asymmetrical & -20.38 & -17.33 & 0.8 & 0.546 \\
\hline \multirow{3}{*}{ Symmetrical } & $76(N=100)$, symmetrical & -23.56 & \multicolumn{2}{|c|}{-20.53 (by GA in [3]) } & - \\
\hline & $78(N=100)$, symmetrical & -22.97 & \multicolumn{2}{|c|}{-20.56 (by GA in [3]) } & - \\
\hline & $80(N=100)$, symmetrical & -22.2 & \multicolumn{2}{|c|}{-20.52 (by ACO in [5]) } & - \\
\hline
\end{tabular}

obtained by the IFT-DE, respectively, shows PSLL decrement about $2.4 \mathrm{~dB}$ and $3.0 \mathrm{~dB}$, as is compared to the value given by GA [3]. Furthermore, for the purpose of comparison, all the above synthesis results using the IFT-DE and that obtained by other tools are tabulated in Table 1. It indicates that through performing the proposed method, the SLA shows about $1 \sim 3 \mathrm{~dB}$ PSLL suppression without a notable tradeoff at the beamwidth. Therefore, the effectiveness of the IFT-DE is confirmed.

All the aforementioned results are obtained by using a PC equipped with an 8 GB RAM as well as an Intel I7-6700 Processor that operates at $3.4 \mathrm{GHz}$, and the hardware cost is about an hour for each numerical instance.

\section{Conclusion}

The use of IFT could efficiently provide a thinned linear array with improved sidelobe performance. For the assumption that the element spacing is not less than half wavelength, most element locations of the thinned array are predetermined after performing the IFT. Therefore, the number of element locations needing to be optimized is largely reduced, which makes the solution space of DE greatly narrowed.
Consequently, the low sidelobe SLA with many elements could be synthesized at relative low hardware cost. Some numerical results confirmed the effectiveness of the IFT-DE in synthesizing large SLA at a faster convergence speed. Furthermore, by simple modification, the proposed method can also be extended to $2 \mathrm{D}$ arrays and thereby provides a candidate way for pattern synthesis of planar sparse arrays.

\section{Data Availability}

The data used to support the findings of this study are available from the corresponding author upon request.

\section{Conflicts of Interest}

The authors declare that they have no conflicts of interest.

\section{Acknowledgments}

This work is supported in part by the National Natural Science Foundation of China under Grant nos. 61601272 and 61772398, in part by the Natural Science Foundation of Shaanxi Province under Grant no. 2016JM6068, in part by 
the scientific research plan of the Education Department of Shaanxi Province under Grant no. 15JK1147, and in part by the scientific research plan of Shaanxi University of Technology under Grant no. SLGQD14-05.

\section{References}

[1] R. J. Mailloux, Phased Array Antenna Handbook, Artech House, Boston, Norwood, MA, 2nd edition, 2005.

[2] R. L. Haupt, "Thinned arrays using genetic algorithms," IEEE Transactions on Antennas and Propagation, vol. 42, no. 7, pp. 993-999, 1994.

[3] G. K. Mahanti, N. Pathak, and P. K. Mahanti, "Synthesis of thinned linear antenna arrays with fixed sidelobe level using real-coded genetic algorithm," Progress In Electromagnetics Research, vol. 75, pp. 319-328, 2007.

[4] L. Zhang, Y. C. Jiao, B. Chen, and H. Li, "Orthogonal genetic algorithm for planar thinned array designs," International Journal of Antennas and Propagation, vol. 2012, Article ID 319037, 7 pages, 2012.

[5] O. Quevedo-Teruel and E. Rajo-Iglesias, "Ant colony optimization in thinned array synthesis with minimum sidelobe level," IEEE Antennas and Wireless Propagation Letters, vol. 5, pp. 349-352, 2006.

[6] Q. Jiang, J. X. Chen, and D. Liu, "Binary inheritance learning particle swarm optimisation and its application in thinned antenna array synthesis with the minimum sidelobe level," IET Microwaves, Antennas \& Propagation, vol. 9, no. 13, pp. 1386-1391, 2015.

[7] S. K. Goudos and J. N. Sahalos, "Design of large thinned arrays using different biogeography-based optimization migration models," International Journal of Antennas and Propagation, vol. 2016, Article ID 5359298, 11 pages, 2016.

[8] W. P. M. N. Keizer, "Linear array thinning using iterative FFT techniques," IEEE Transactions on Antennas and Propagation, vol. 56, no. 8, pp. 2757-2760, 2008.

[9] W. Keizer, "Large planar array thinning using iterative FFT techniques," IEEE Transactions on Antennas and Propagation, vol. 57, no. 10, pp. 3359-3362, 2009.

[10] X. K. Wang, Y. C. Jiao, and Y. Y. Tan, "Gradual thinning synthesis for linear array based on iterative Fourier techniques," Progress In Electromagnetics Research, vol. 123, pp. 299-320, 2012.

[11] G. Oliveri, L. Manica, and A. Massa, "ADS-based guidelines for thinned planar arrays," IEEE Transactions on Antennas and Propagation, vol. 58, no. 6, pp. 1935-1948, 2010.

[12] A. Trucco and V. Murino, "Stochastic optimization of linear sparse arrays," IEEE Journal of Oceanic Engineering, vol. 24, no. 3, pp. 291-299, 1999.

[13] D. Liu, Q. Feng, W. B. Wang, and X. Yu, "Synthesis of unequally spaced antenna arrays by using inheritance learning particle swarm optimization," Progress In Electromagnetics Research, vol. 118, pp. 205-221, 2011.

[14] D. G. Kurup, M. Himdi, and A. Rydberg, "Synthesis of uniform amplitude unequally spaced antenna arrays using the differential evolution algorithm," IEEE Transactions on Antennas and Propagation, vol. 51, no. 9, pp. 2210-2217, 2003.

[15] S. K. Goudos, K. Siakavara, T. Samaras, E. E. Vafiadis, and J. N. Sahalos, "Sparse linear array synthesis with multiple constraints using differential evolution with strategy adaptation," IEEE Antennas and Wireless Propagation Letters, vol. 10, pp. 670-673, 2011.

[16] K. Chen, H. Chen, L. Wang, and H. Wu, "Modified real GA for the synthesis of sparse planar circular arrays," IEEE Antennas and Wireless Propagation Letters, vol. 15, pp. 274-277, 2016.

[17] O. M. Bucci and S. perna, "A deterministic two dimensional density taper approach for fast design of uniform amplitude pencil beams arrays," IEEE Transactions on Antennas and Propagation, vol. 59, no. 8, pp. 2852-2861, 2011.

[18] Y. Liu, Z. Nie, and Q. H. Liu, "Reducing the number of elements in a linear antenna array by the matrix pencil method," IEEE Transactions on Antennas and Propagation, vol. 56, no. 9, pp. 2955-2962, 2008.

[19] G. Oliveri and A. Massa, "Bayesian compressive sampling for pattern synthesis with maximally sparse non-uniform linear arrays," IEEE Transactions on Antennas and Propagation, vol. 59, no. 2, pp. 467-481, 2011.

[20] P. Rocca, G. Oliveri, R. J. Mailloux, and A. Massa, "Unconventional phased array architectures and design methodologies-a review," Proceedings of the IEEE, vol. 104, no. 3, pp. 544-560, 2016.

[21] G. Oliveri, M. Carlin, and A. Massa, "Complex-weight sparse linear array synthesis by Bayesian compressive sampling," IEEE Transactions on Antennas and Propagation, vol. 60, no. 5, pp. 2309-2326, 2012.

[22] P. Rocca, G. Oliveri, and A. Massa, "Differential evolution as applied to electromagnetics," IEEE Antennas and Propagation Magazine, vol. 53, no. 1, pp. 38-49, 2011. 


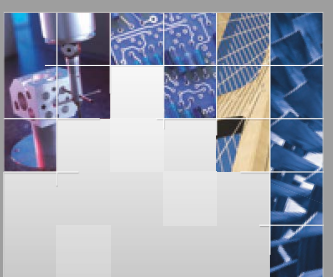

\section{Enfincering}
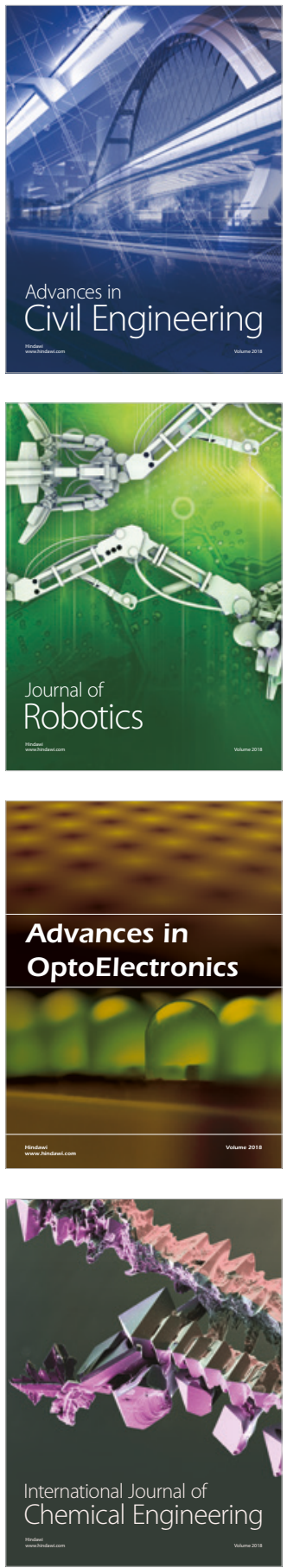

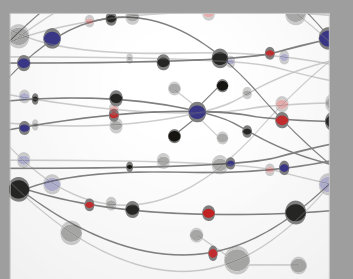

\section{Rotating \\ Machinery}

The Scientific World Journal

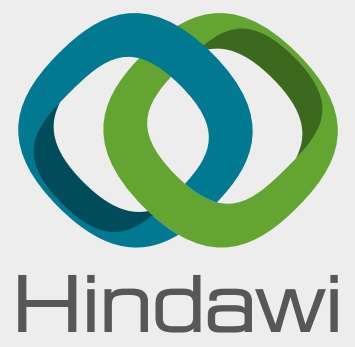

Submit your manuscripts at

www.hindawi.com
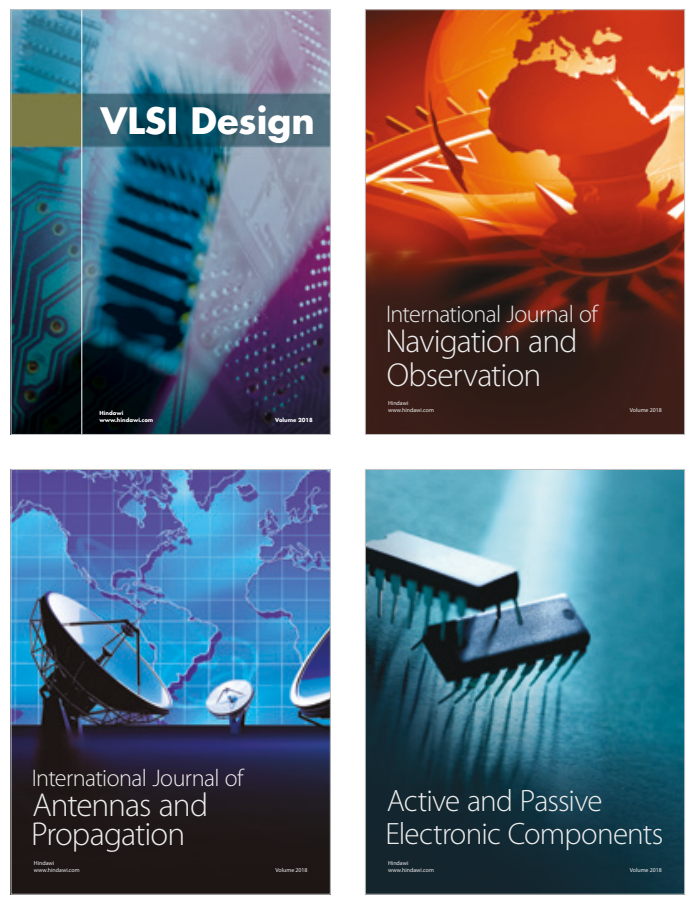
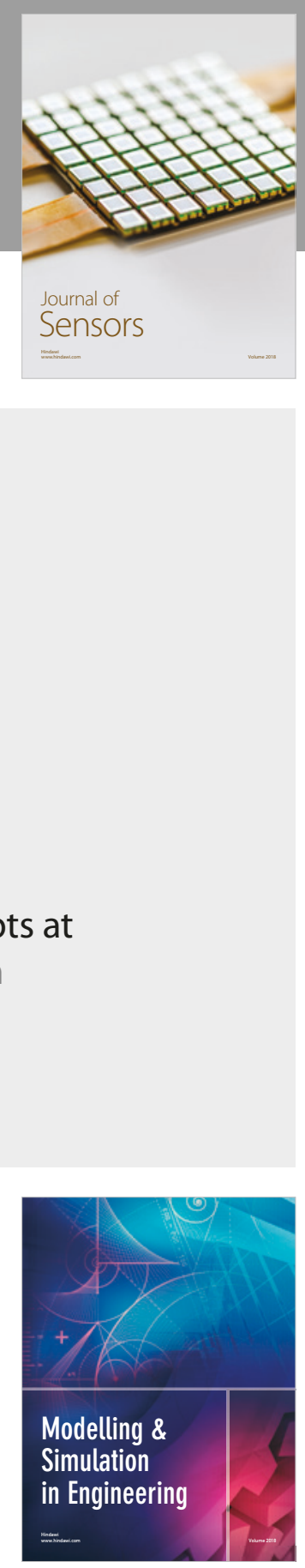

\section{Advances \\ Multimedia}
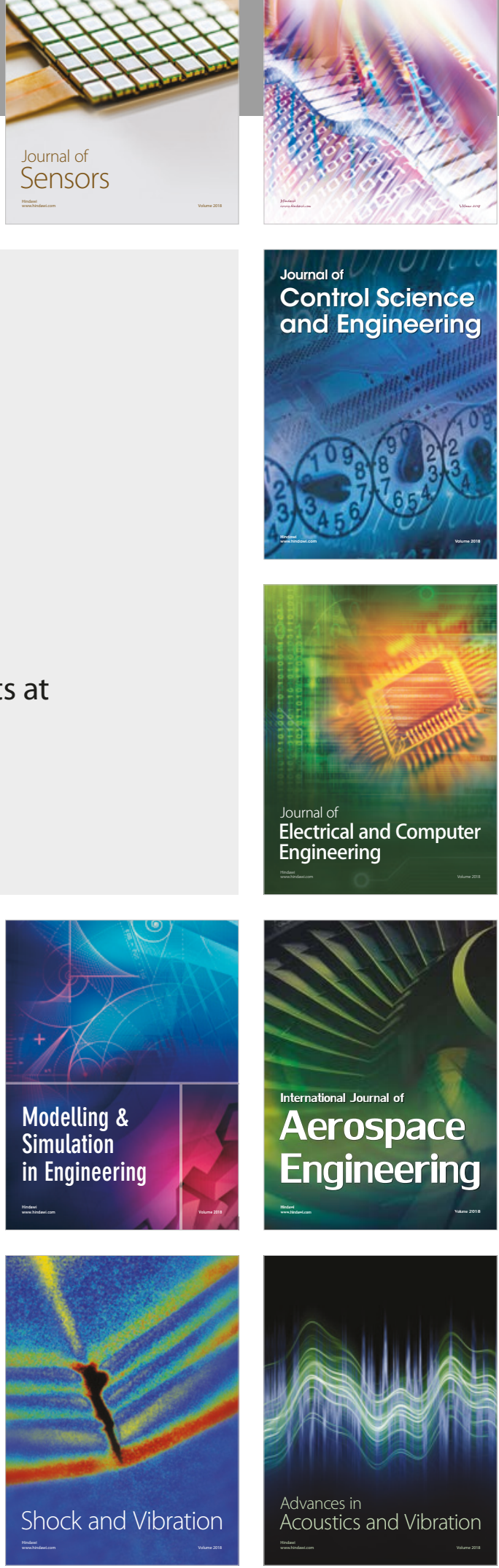